\title{
ON LATTICE-PERFECT MEASURES
}

\author{
P. D. STRATIGOS \\ (Received 5 February 1990; revised 15 August 1991)
}

Communicated by J. H. Rubinstein

\begin{abstract}
The general properties of lattice-perfect measures are discussed. The relationship between countable compactness and measure perfectness, and the relationship between lattice-measure tightness and lattice-measure perfectness are investigated and several applications in topological measure theory are given.

1991 Mathematics subject classification (Amer. Math. Soc.): 28 A 60, 28 A 33, 28 C 15.

Keywords and phrases: Lattice space; countable compactness; regular, $\sigma$-smooth, or tight lattice measure; strongly measure repleteness; lattice-perfect measure; characteristic function of a sequence of sets.
\end{abstract}

\section{Introduction}

The present paper is devoted to lattice-perfect measures. It was motivated by Sazonov's paper, "On perfect measures" [9]. The concept of perfect measure was first defined in the book [5] by Gnedenko and Kolmogorov. This concept has important applications in probability theory. A discussion of this fact is given in Sazonov [9, Introduction, part 3].

In his paper, Sazonov discusses the general properties of perfect measures. He also discusses the relationship between countable compactness and measure perfectness, and the relationship between measure tightness and measure perfectness in topological spaces.

In the present paper, the definition of perfect measure, as given by Sazonov,

(C) 1994 Australian Mathematical Society 0263-6115/94 \$A2.00+0.00 
is generalized. This generalization involves two arbitrary lattices. (For this reason, the generalized concept of perfect measure will be referred to as latticeperfect measure). As a result, generalizations of several results of Sazonov are obtained.

More specifically, in Section 2, several 'carry over' properties of latticemeasure perfectness are discovered. In Section 3, the relationship between countable compactness and measure perfectness is investigated, without using the concept of characteristic function of a sequence of sets, as is done in Sazonov's paper, but by using a direct approach, which is in the spirit of Alexandroff, namely, an approach which is based on an abstract Lusin-type theorem. In Section 4, the relationship between lattice-measure tightness and lattice-measure perfectness is investigated.

Several applications in topological measure theory are given throughout the paper.

We note that the generalizations mentioned above are important for applications in probability theory, where one considers random functions (transformations), rather than random variables. We will discuss such applications elsewhere, in order to remain within the scope of the present paper.

\section{Terminology and notation}

The terminology and notation are fairly standard and are consistent with those of Wallman [12], Alexandroff [1], Frolik [4], Nöbeling [7], Bachman and Sultan [2], as well as Szeto [10] and Grassi [6].

For the convenience of the reader, in this section we will present some of the special terminology and notation which is used throughout the paper.

(a) Consider any set $X$ and any lattice $\mathscr{L}$ on $X$.

We shall always assume, without loss of generality for our purposes, that $\emptyset, X \in \mathscr{L}$.

Now, consider any topological space $X$ and denote the class of open sets by $\mathscr{U}$, the class of closed sets by $\mathscr{F}$, and the class of zero sets by $\mathscr{Z}$. Note each of the classes $\mathscr{U}, \mathscr{F}, \mathscr{Z}$ is a lattice of the prescribed type. Recall $\mathscr{U}$ is also referred to as the topology on $X$ and the topological space $X$ is defined to be $\langle X, \mathscr{U}\rangle$. Thus $\langle X, \mathscr{L}\rangle$ is a generalization of a topological space. For this reason, we shall refer to $\langle X, \mathscr{L}\rangle$ as a lattice space. In topological measure theory, it is convenient to regard $\mathscr{F}$ as the topology on $X$ and $\langle X, \mathscr{F}\rangle$ as the topological space.

The definitions of the following concepts are found in [2]: $\mathscr{L}$ is $\delta$, complement 
generated, separating, disjunctive, normal, countably compact; a subset of $X$ is $\mathscr{L}$-compact.

(b) A function $f$ from $X$ to $\mathrm{R} \cup\{ \pm \infty\}$ is said to be $\mathscr{L}$-continuous if and only if for every closed set $C$ of $\mathrm{R} \cup\{ \pm \infty\}, f^{-1}(C) \in \mathscr{L}$. The set whose general element is a function from $X$ to $\mathrm{R} \cup\{ \pm \infty\}$ which is $\mathscr{L}$-continuous and bounded is denoted by $C_{b}(\mathscr{L})$.

The set whose general element is the intersection of an arbitrary subset of $\mathscr{L}$ is denoted by $t \mathscr{L}$. The algebra of subsets of $X$ generated by $\mathscr{L}$ is denoted by $\mathscr{A}(\mathscr{L})$. The $\sigma$-algebra of subsets of $X$ generated by $\mathscr{L}$ is denoted by $\sigma(\mathscr{L})$. The class of subsets of $X$ which is closed under the formation of countable unions and intersections, contains $\mathscr{L}$, and is minimal, is denoted by $\rho(\mathscr{L})$. The class of subsets of $X$ obtainable by the lattice-Souslin operations is denoted by $s(\mathscr{L})$.

(c) Consider any algebra $\mathscr{A}$ on $X$. A measure on $\mathscr{A}$ is defined to be a function $\mu$, from $\mathscr{A}$ to $\mathrm{R}$, such that $\mu$ is finitely additive and bounded. (See [1, page 567]). The set whose general element is a measure on $\mathscr{A}(\mathscr{L})$ is denoted by $M(\mathscr{L})$. An element $\mu \in M(\mathscr{L})$ is said to be $\mathscr{L}$-regular if and only if for every element $E \in \mathscr{A}(\mathscr{L})$, for every positive number $\epsilon$, there exists an element $L \in \mathscr{L}$ such that $L \subset E$ and $|\mu(E)-\mu(L)|<\epsilon$. The set whose general element is an element $\mu \in M(\mathscr{L})$ which is $\mathscr{L}$-regular is denoted by $M_{\mathrm{R}}(\mathscr{L})$. An element $\mu \in M(\mathscr{L})$ is said to be $\mathscr{L}$ - ( $\sigma$-smooth) if and only if for every sequence in $\mathscr{A}(\mathscr{L}),\left\langle A_{n}\right\rangle$, if $\left\langle A_{n}\right\rangle$ is decreasing and $\lim _{n} A_{n}=\emptyset$, then $\lim _{n} \mu\left(A_{n}\right)=0$. The set whose general element is an element of $M(\mathscr{L})$ which is $\mathscr{L}$ - $(\sigma$-smooth) is denoted by $M^{\sigma}(\mathscr{L})$. An element $\mu \in M(\mathscr{L})$ is said to be $\mathscr{L}$-tight if and only if $\mu \in M^{\sigma}(\mathscr{L})$ and for every positive number $\epsilon$, there exists an $\mathscr{L}$-compact set $K$ such that $|\mu|_{*}\left(K^{\prime}\right)<\epsilon$. The set whose general element is an element of $M(\mathscr{L})$ which is $\mathscr{L}$-tight is denoted by $M^{t}(\mathscr{L})$.

$\mathscr{L}$ is said to be strongly measure replete if and only if $M_{\mathrm{R}}^{\sigma}(\mathscr{L})=M_{\mathrm{R}}^{t}(\mathscr{L})$.

NOTE. Since every element of $M(\mathscr{L})$ is expressible as the difference of nonnegative elements of $M(\mathscr{L})$, without loss of generality, we shall work with nonnegative elements of $M(\mathscr{L})$.

\section{Lattice-perfect measures and some of their properties}

In this section, the definition of perfect measure given by Sazonov is generalised, by introducing the concept of lattice-perfect measure. Several 'carry-over' 
properties of lattice-measure perfectness are discovered and applications are given whenever an opportunity arises.

In his paper, Sazonov defines the concept of perfect measure as follows: Consider any measure space $\langle X, \mathscr{S}, \mu\rangle$ such that $\mathscr{S}$ is a $\sigma$-algebra and $\mu$ is finite. Sazonov calls $\mu$ perfect if and only if for every $\mathscr{S}$-measurable function $f$ from $X$ to $\mathrm{R}$ and for every subset $E$ of $\mathrm{R}$ such that $f^{-1}(E) \in \mathscr{S}$, there exists a Borel set $B$ of $\mathrm{R}$, such that $B \subset E$ and $\mu\left(f^{-1}(B)\right)=\mu\left(f^{-1}(E)\right)$.

OBSERVATION. Denote the class of R-closed sets by $\mathscr{F}$. Note $\mathscr{F}$ is a lattice and the class of Borel sets of $\mathrm{R}$ is $\sigma(\mathscr{F})$.

This observation leads us to generalize Sazonov's concept of perfect measure as follows: Consider any lattice space $\left(X, \mathscr{L}_{1}\right)$ and any element of $M^{\sigma}\left(\mathscr{L}_{1}\right)$. The following statement is true: there exists a unique countably additive, finite measure on $\sigma\left(\mathscr{L}_{1}\right)$ which is an extension of $\mu$. This measure is also denoted by $\mu$. Thus there exists a one-to-one correspondence between $M^{\sigma}\left(\mathscr{L}_{1}\right)$ and the set whose general element is a countably additive, finite measure on $\sigma\left(\mathscr{L}_{1}\right)$. Further, consider any other lattice space $\left\langle Y, \mathscr{L}_{2}\right\rangle$.

Definition 2.1. An element $\mu \in M^{\sigma}\left(\mathscr{L}_{1}\right)$ is $\left\langle\mathscr{L}_{1}, \mathscr{L}_{2}\right\rangle$-perfect if and only if for every $\left\langle\sigma\left(\mathscr{L}_{1}\right), \sigma\left(\mathscr{L}_{2}\right)\right\rangle$-measurable function $T$ from $X$ to $Y$ and for every subset $E$ of $Y$ such that $T^{-1}(E) \in \sigma\left(\mathscr{L}_{1}\right)$, there exists an element $B \in \sigma\left(\mathscr{L}_{2}\right)$ such that $B \subset E$ and $\mu\left(T^{-1}(B)\right)=\mu\left(T^{-1}(E)\right)$.

We note that this generalization is important for applications in probability theory, where one considers random functions (transformations), rather than random variables.

The following generalizes various results of Sazonov [9].

LEMMA 2.2. Consider the setting of Definition 2.1. An element $\mu \in M^{\sigma}\left(\mathscr{L}_{1}\right)$ is $\left\langle\mathscr{L}_{1}, \mathscr{L}_{2}\right\rangle$-perfect if and only if the following condition is satisfied:

for every $\left\langle\sigma\left(\mathscr{L}_{1}\right), \sigma\left(\mathscr{L}_{2}\right)\right\rangle$-measurable function $T$ from $X$ to $Y$, there exists an element $B \in \sigma\left(\mathscr{L}_{2}\right)$ such that

$$
B \subset T(X) \quad \text { and } \quad \mu\left(T^{-1}(B)\right)=\mu(X) .
$$

Proof. Consider any element $\mu \in M^{\sigma}\left(\mathscr{L}_{1}\right)$.

(i) Assume $\mu$ is $\left\langle\mathscr{L}_{1}, \mathscr{L}_{2}\right\rangle$-perfect. Consider any $\left\langle\sigma\left(\mathscr{L}_{1}\right), \sigma\left(\mathscr{L}_{2}\right)\right\rangle$-measurable function $T$ from $X$ to $Y$. Then since $\mu$ is $\left\langle\mathscr{L}_{1}, \mathscr{L}_{2}\right\rangle$-perfect, $T(X) \subset Y$ and $T^{-1}(T(X))=X \in \sigma\left(\mathscr{L}_{1}\right)$, by the relevant definition, there exists an element 
$B \in \sigma\left(\mathscr{L}_{2}\right)$ such that $B \subset T(X)$ and $\mu\left(T^{-1}(B)\right)=\mu\left(T^{-1}(T(X))\right)$. Consider any such $B$. Note $\mu\left(T^{-1}(B)\right)=\mu(X)$. Thus condition (*) is satisfied.

(ii) Assume condition (*) is satisfied. To show $\mu$ is $\left\langle\mathscr{L}_{1}, \mathscr{L}_{2}\right\rangle$-perfect, use the relevant definition, namely, consider any function $T$ from $X$ to $Y$ such that $T$ is $\left\langle\sigma\left(\mathscr{L}_{1}\right), \sigma\left(\mathscr{L}_{2}\right)\right\rangle$-measurable, and any subset $E$ of $Y$ such that $T^{-1}(E) \in$ $\sigma\left(\mathscr{L}_{1}\right)$, and show there exists an element $B \in \sigma\left(\mathscr{L}_{2}\right)$ such that $B \subset E$ and $\mu\left(T^{-1}(B)\right)=\mu\left(T^{-1}(E)\right)$.

Case I. $T^{-1}(E)=\emptyset$. Then let $B=\emptyset$.

Case II. $T^{-1}(E) \neq \emptyset$. Then denote the general element of $X$ by $x$ and consider any element $y_{1}$ of $T\left(T^{-1}(E)\right)$. Now, consider the function $\tilde{T}$ determined by

$$
\tilde{T}(x)= \begin{cases}T(x) & \text { if } x \in T^{-1}(E), \\ y_{1} & \text { if } x \in C T^{-1}(E) .\end{cases}
$$

Note $\tilde{T}$ is $\left\langle\sigma\left(\mathscr{L}_{1}\right), \sigma\left(\mathscr{L}_{2}\right)\right\rangle$-measurable. Then, since condition $(*)$ is satisfied, there exists an element $B \in \sigma\left(\mathscr{L}_{2}\right)$ such that $B \subset \tilde{T}(X)$ and $\mu\left(\tilde{T}^{-1}(B)\right)=$ $\mu(X)$. Consider any such $B$. Then $B \subset E$ and $\mu\left(T^{-1}(B)\right)=\mu\left(T^{-1}(E)\right)$. Consequently $\mu$ is $\left\langle\mathscr{L}_{1}, \mathscr{L}_{2}\right\rangle$-perfect.

NOTE. Condition $(*)$ is implied by the following condition:

For every $\left\langle\sigma\left(\mathscr{L}_{1}\right), \sigma\left(\mathscr{L}_{2}\right)\right\rangle$-measurable function $T$ from $X$ to $Y$, and for every positive number $\epsilon$, there exists an element $L_{2} \in \mathscr{L}_{2}$ such that

$$
L_{2} \subset T(X) \quad \text { and } \quad \mu\left(T^{-1}\left(L_{2}\right)\right)>\mu(X)-\epsilon .
$$

Next, consider the following: any lattice space $\left\langle X, \mathscr{L}_{1}\right\rangle$; any element $\mu \in$ $M^{\sigma}\left(\mathscr{L}_{1}\right)$, and the measure space $\left\langle X, \sigma\left(\mathscr{L}_{1}\right), \mu\right\rangle$; any subset $X_{1}$ of $X$, and the function $\phi$ on $X_{1} \cap \sigma\left(\mathscr{L}_{1}\right)$ determined by $\phi(E)=\inf \left\{\mu(A) \mid A \in \sigma\left(\mathscr{L}_{1}\right)\right.$ and $A \supset E\}$. Then $\phi$ is a measure, referred to as the measure on $X_{1} \cap \sigma\left(\mathscr{L}_{1}\right)$ induced by $\mu$, and denoted by $\mu_{X_{1}}$.

THEOREM 2.3. Consider the setting described above, in conjunction with the setting of Definition 2.1. If $\mu$ is $\left\langle\mathscr{L}_{1}, \mathscr{L}_{2}\right\rangle$-perfect, $X_{1} \in \sigma\left(\mathscr{L}_{1}\right)$, and $\sigma\left(\mathscr{L}_{2}\right)$ contains a singleton, then $\mu_{X_{1}}$ is $\left\langle X_{1} \cap \mathscr{L}_{1}, \mathscr{L}_{2}\right\rangle$-perfect.

Proof. Assume $\mu$ is $\left\langle\mathscr{L}_{1}, \mathscr{L}_{2}\right\rangle$-perfect, $X_{1} \in \sigma\left(\mathscr{L}_{1}\right)$ and $\sigma\left(\mathscr{L}_{2}\right)$ contains a singleton. To show $\mu_{X_{1}}$ is $\left\langle X_{1} \cap \mathscr{L}_{1}, \mathscr{L}_{2}\right\rangle$-perfect, use Lemma 2.2, namely, show condition (*) is satisfied. Accordingly, consider any $\left\langle X_{1} \cap \sigma\left(\mathscr{L}_{1}\right), \sigma\left(\mathscr{L}_{2}\right)\right\rangle$ measurable function $T_{1}$ from $X_{1}$ to $Y$ and show there exists an element $B_{1} \in$ $\sigma\left(\mathscr{L}_{2}\right)$ such that $B_{1} \subset T_{1}\left(X_{1}\right)$ and $\mu_{X_{1}}\left(T_{1}^{-1}\left(B_{1}\right)\right)=\mu_{X_{1}}\left(X_{1}\right)$. 
Denote the general element of $X$ by $x$ and consider any element $w$ of $Y$ such that $\{w\} \in \sigma\left(\mathscr{L}_{2}\right)$, using the assumption. Now, consider the function $T$ determined by

$$
T(x)=\left\{\begin{array}{ll}
T_{1}(x) & \text { if } x \in X_{1} \\
w & \text { if } x \in C X_{1}
\end{array} .\right.
$$

Note $T$ is $\left\langle\sigma\left(\mathscr{L}_{1}\right), \sigma\left(\mathscr{L}_{2}\right)\right\rangle$-measurable. Hence since $\mu$ is $\left\langle\mathscr{L}_{1}, \mathscr{L}_{2}\right\rangle$-perfect, by Lemma 2.2 , there exists an element $B \in \sigma\left(\mathscr{L}_{2}\right)$ such that $B \subset T(X)$ and $\mu\left(T^{-1}(B)\right)=\mu(X)$. Consider any such $B$. Now, consider the subset of $Y, B_{1}$, determined by

$$
B_{1}= \begin{cases}B & \text { if } w \in T_{1}\left(X_{1}\right), \\ B-\{w\} & \text { if } w \in C T_{1}\left(X_{1}\right) .\end{cases}
$$

Then $B_{1} \in \sigma\left(\mathscr{L}_{2}\right)$ and $B_{1} \subset T_{1}\left(X_{1}\right)$ and $\mu_{X_{1}}\left(T_{1}^{-1}\left(B_{1}\right)\right)=\mu_{X_{1}}\left(X_{1}\right)$. Consequently $\mu_{X_{1}}$ is $\left(X_{1} \cap \mathscr{L}_{1}, \mathscr{L}_{2}\right\rangle$-perfect.

NOTE. The following statement is also true, with a similar proof: if $\mu$ is $\left\langle\mathscr{L}_{1}, \mathscr{L}_{2}\right\rangle$-perfect and $X_{1} \in \sigma\left(\mathscr{L}_{1}\right)$ and for every $\left\langle X_{1} \cap \sigma\left(\mathscr{L}_{1}\right), \sigma\left(\mathscr{L}_{2}\right)\right\rangle$ measurable function $T_{1}$ from $X_{1}$ to $Y, T_{1}\left(X_{1}\right) \in \sigma\left(\mathscr{L}_{2}\right)$, then $\mu_{X_{1}}$ is $\left\langle X_{1} \cap\right.$ $\left.\mathscr{L}_{1}, \mathscr{L}_{2}\right\rangle$-perfect.

APPLICATION 2.4. Consider the following: any lattice space $\left\langle X, \mathscr{L}_{1}\right\rangle$, any topological space $\left\langle Y, \mathscr{F}_{2}\right\rangle$ such that $\mathscr{F}_{2}$ is $T_{1}$, any element $\mu \in M^{\sigma}\left(\mathscr{L}_{1}\right)$ such that $\mu$ is $\left\langle\mathscr{L}_{1}, \mathscr{F}_{2}\right\rangle$-perfect, any element $X_{1} \in \sigma\left(\mathscr{L}_{1}\right)$, and the measure space $\left\langle X_{1}, X_{1} \cap \sigma\left(\mathscr{L}_{1}\right), \mu_{X_{1}}\right\rangle$. Then, by Theorem $2.3, \mu_{X_{1}}$ is $\left\langle X_{1} \cap \mathscr{L}_{1}, \mathscr{F}_{2}\right\rangle$-perfect.

THEOREM 2.5. Consider the following: any measurable space $\langle X, \mathscr{S}\rangle$ such that $\mathscr{S}$ is a $\sigma$-algebra, any topological space $\left\langle Y, \mathscr{F}_{2}\right\rangle$, any element $\mu \in$ $M^{\sigma}(\mathscr{S})$, the measure space $\langle X, \mathscr{S}, \mu\rangle$, and its completion $\langle X, \hat{\mathscr{S}}, \hat{\mu}\rangle$. Assume $\mathscr{F}_{2}$ is $T_{1}$ and $\mathscr{F}_{2}$ satisfies the Second Axiom of Countability. Then $\mu$ being $\left\langle\mathscr{S}, \mathscr{F}_{2}\right\rangle$-perfect implies $\hat{\mu}$ is $\left\langle\hat{\mathscr{S}}, \mathscr{F}_{2}\right\rangle$-perfect.

Proof. Assume $\mu$ is $\left\langle\mathscr{S}, \mathscr{F}_{2}\right\rangle$-perfect. To show $\hat{\mu}$ is $\left\langle\hat{\mathscr{S}}, \mathscr{F}_{2}\right\rangle$-perfect, use Lemma 2.2 , namely, show condition (*) is satisfied. Accordingly, consider any $\left\langle\hat{\mathscr{S}}, \sigma\left(\mathscr{F}_{2}\right)\right\rangle$-measurable function $\hat{T}$ from $X$ to $Y$, and show there exists an element $B \in \sigma\left(\mathscr{F}_{2}\right)$ such that $B \subset \hat{T}(X)$ and $\hat{\mu}\left(\hat{T}^{-1}(B)\right)=\hat{\mu}(X)$.

Step I. Show there exists a function $T$ from $X$ to $Y$, which is $\left\langle\mathscr{S}, \sigma\left(\mathscr{F}_{2}\right)\right\rangle$ -measurable, such that $T=\hat{T}$ a.e. $(\hat{\mu})$.

Consider any countable base $\mathscr{F}_{2}^{*}$ for $\mathscr{F}_{2}$, using the assumption. Set $\mathscr{F}_{2}^{*}=$ $\left\{E_{k} ; k \in N^{*}\right\}$. 
Note that since $\hat{T}$ is $\left\langle\hat{\mathscr{S}}, \sigma\left(\mathscr{F}_{2}\right)\right\rangle$-measurable, $\hat{T}^{-1}\left(E_{k}\right) \in \hat{\mathscr{S}}$ for every $k$. Hence there exists an element $S_{k} \in \mathscr{S}$, and an element $N_{k} \in \mathscr{P}(X)$ such that $\hat{T}^{-1}\left(E_{k}\right)=S_{k} \cup N_{k}$, and there exists an element $W_{k} \in \mathscr{S}$ such that $\mu\left(W_{k}\right)=0$ and $W_{k} \supset N_{k}$. Consider any such $S_{k}, N_{k}, W_{k}$.

Consider $\bigcup_{k} W_{k}$. Note $\bigcup_{k} W_{k} \in \mathscr{S}$. Set $\bigcup_{k} W_{k}=V$. Denote the general element of $X$ by $x$ and consider any element $b$ of $Y$. Now, consider the function $T$ determined by

$$
T(x)= \begin{cases}b & \text { if } x \in V, \\ \hat{T}(x) & \text { if } x \in C V .\end{cases}
$$

Then $T$ is a function from $X$ to $Y$ which is $\left\langle\mathscr{S}, \sigma\left(\mathscr{F}_{2}\right)\right\rangle$-measurable and such that $T=\hat{T}$ a.e. $(\hat{\mu})$.

Step II. Note $C V \in \mathscr{S}$ and $C V \subset\{x \mid T(x)=\hat{T}(x)\}$ and $\mu(C V)=\mu(X)$. Set $C V=X_{1}$ and consider the measure space $\left\langle X_{1}, X_{1} \cap \mathscr{S}, \mu_{X_{1}}\right\rangle$. Then, by Theorem 2.3, $\mu_{X_{1}}$ is $\left\langle X_{1} \cap \mathscr{S}, \mathscr{F}_{2}\right\rangle$-perfect. Now, consider $\left.T\right|_{X_{1}}$. Note since $T$ is $\left\langle\mathscr{S}, \sigma\left(\mathscr{F}_{2}\right)\right\rangle$-measurable and $X_{1} \in \mathscr{S},\left.T\right|_{X_{1}}$ is $\left\langle X_{1} \cap \mathscr{S}, \sigma\left(\mathscr{F}_{2}\right)\right\rangle$-measurable. Set $\left.T\right|_{X_{1}}=T_{1}$. Note since $\mu_{X_{1}}$ is $\left\langle X_{1} \cap \mathscr{S}, \mathscr{F}_{2}\right\rangle$-perfect, by Lemma 2.2 , there exists an element $B \in \sigma\left(\mathscr{F}_{2}\right)$ such that $B \subset T_{1}\left(X_{1}\right)$ and $\mu_{X_{1}}\left(T_{1}^{-1}(B)\right)=$ $\mu_{X_{1}}\left(X_{1}\right)$. Consider any such $B$. Then $B \subset \hat{T}(X)$ and $\hat{\mu}\left(\hat{T}^{-1}(B)\right)=\hat{\mu}(X)$. Consequently $\hat{\mu}$ is $\left\langle\hat{\mathscr{S}}, \mathscr{F}_{2}\right\rangle$-perfect.

NOTE. The statement that $\hat{\mu}$ is $\left\langle\hat{\mathscr{S}}, \mathscr{F}_{2}\right\rangle$-perfect implies $\mu$ is $\left\langle\mathscr{S}, \mathscr{F}_{2}\right\rangle$-perfect is true, in general.

\section{Countable compactness and measure perfectness}

In this section, the relationship between countable compactness and measure perfectness is investigated, without using the concept of characteristic function of a sequence of sets, as is done in Sazonov's paper, but by using a direct approach, which is in the spirit of Alexandroff, namely, an approach which is based on an abstract Lusin-type theorem. Several applications are given.

LEMMA 3.1. Consider the following: any lattice space $\langle X, \mathscr{L}\rangle$ such that $\mathscr{L}$ is $\delta$; the vector space $C_{b}(\mathscr{L})$, the sup norm on $C_{b}(\mathscr{L})$, the norm derived metric $d$ on $C_{b}(\mathscr{L})$, and the metric space $\left\langle C_{b}(\mathscr{L}), d\right\rangle$. Then $\left\langle C_{b}(\mathscr{L}), d\right\rangle$ is complete.

A proof outline will be given for the convenience of the reader. 
Consider any Cauchy sequence $\left\langle f_{n}\right\rangle$ in $C_{b}(\mathscr{L})$, and show it is convergent, that is, that there exists an element $g \in C_{b}(\mathscr{L})$ such that $\lim _{n} f_{n}=g$.

Denote the general element of $X$ by $x$ and consider $\left\langle f_{n}(x)\right\rangle$. Note for every $x,\left\langle f_{n}(x)\right\rangle$ is Cauchy. Hence for every $x$, since $\mathbf{R}^{1}$ is complete, $\left\langle f_{n}(x)\right\rangle$ is convergent. Consider the function $g$ which is such that $D_{g}=X$ and for every $x, g(x)=\lim _{n} f_{n}(x)$. Show $g \in C_{b}(\mathscr{L})$.

I. Show $g \in C(\mathscr{L})$. For this, show the convergence of $\left\langle f_{n}(x)\right\rangle$ to $g(x)$ is uniform and then use the assumption that $\mathscr{L}$ is $\delta$.

II. Show $g$ is bounded. For this, use the fact that the convergence of $\left\langle f_{n}(x)\right\rangle$ to $g(x)$ is uniform.

LEMMA 3.2. Consider any lattice space $\langle X, \mathscr{L}\rangle$. Let $\mu \in M^{\sigma}(\mathscr{L})$. If $\mathscr{L}$ is $\delta$ and $\mu \in M_{\mathrm{R}}^{\sigma}(\mathscr{L})$, then for every $\sigma(\mathscr{L})$-measurable set $E$, for every $\sigma(\mathscr{L})$ measurable function $f$ from $E$ to $R$, for every positive number $\epsilon$, there exists an element $D \in \mathscr{L}$ such that $D \subset E,\left.f\right|_{D} \in C_{b}(\mathscr{L})$ and $\mu(E-D)<\epsilon$.

PROOF. Assume $\mathscr{L}$ is $\delta$ and $\mu \in M_{\mathrm{R}}^{\sigma}(\mathscr{L})$. Consider the measurable space $\langle X, \sigma(\mathscr{L})\rangle$ and the extension of $\mu$ to $\sigma(\mathscr{L})$. Denote the extension of $\mu$ to $\sigma(\mathscr{L})$ by $v$. Note since $\mu(X)<+\infty, v(X)<+\infty$ and since $\mu$ is $\mathscr{L}$-regular and $\mathscr{L}$ is $\delta, v$ is $\mathscr{L}$-regular.

Further, consider any $\sigma(\mathscr{L})$-measurable set $E$, any $\sigma(\mathscr{L})$-measurable function $f$ from $E$ to $\mathrm{R}$, and any positive number $\epsilon$.

1. Since $f \in \mathrm{R}^{E}$ and $f$ is $\sigma(\mathscr{L})$-measurable, there exists a sequence $\left\langle g_{n}\right\rangle$ in $\mathrm{R}^{E}$ such that for every $n, g_{n}$ is simple and $\sigma(\mathscr{L})$-measurable, and $\lim _{n} g_{n}=f$, pointwise. Consider any such $\left\langle g_{n}\right\rangle$.

2. Since (a) $v(E)<+\infty$, (b) $f \in \mathbf{R}^{E}$, (c) $f$ is $\sigma(\mathscr{L})$-measurable, (d) for every $n, g_{n} \in \mathrm{R}^{E}$, (e) $g_{n}$ is $\sigma(\mathscr{L})$-measurable, and (f) $\lim _{n} g_{n}=f$, pointwise, by Egoroff's Theorem, there exists an element $A \in \sigma(\mathscr{L})$ such that $A \subset E$, the convergence of $\left\langle g_{n}\right\rangle$ to $f$ is uniform on $A$, and $v(E-A)<\epsilon / 8$ or, equivalently, $v(E)<v(A)+\epsilon / 8$. Consider any such $A$. Then since $v$ is $\mathscr{L}$-regular, there exists an element $B \in \mathscr{L}$ such that $B \subset A$ and $\nu(A)<\nu(B)+\epsilon / 8$.

Consider any such $B$. Then $v(E)<v(B)+\epsilon / 4$ or, equivalently, $v(E-B)<$ $\epsilon / 4$. Set $B=L_{0}$. Thus $L_{0} \in \mathscr{L}, L \subset E$, the convergence of $\left\langle g_{n}\right\rangle$ to $f$ is uniform on $L_{0}$, and $v\left(E-L_{0}\right)<\epsilon / 4$.

3. Observation. Consider any element $h$ of $\mathrm{R}^{E}$, such that $h$ is simple and $\sigma(\mathscr{L})$-measurable. Then there exists an element $\hat{L} \in \mathscr{L}$ such that $\hat{L} \subset E$, $\left.h\right|_{\hat{L}} \in C_{b}(\mathscr{L})$ and $v(E-\hat{L})<\epsilon$.

Now, use this observation on $\left\langle g_{n}\right\rangle$; namely, note for every $n$, since $g_{n} \in \mathrm{R}^{E}$ and $g_{n}$ is simple and $\sigma(\mathscr{L})$-measurable, there exists an element $L_{n} \in \mathscr{L}$, such 
that $L_{n} \subset E,\left.g_{n}\right|_{L_{n}} \in C_{b}(\mathscr{L})$ and $v\left(E-L_{n}\right)<\epsilon / 2^{n+1}$; consider any such $L_{n}$.

4. Consider $\bigcap_{m=0}^{\infty} L_{m}$. Note since $\mathscr{L}$ is $\delta$ and $\left\langle L_{m}\right\rangle$ is in $\mathscr{L}, \bigcap_{m=0}^{\infty} L_{m} \in \mathscr{L}$. Set $\bigcap_{m=0}^{\infty} L_{m}=D$. Then $D \subset E,\left.f\right|_{D} \in C_{b}(\mathscr{L})$ and $\nu(E-D)<\epsilon$.

Now, replace $\nu$ by $\mu$, as usual.

REMARK. This theorem is a generalization of Lusin's Theorem for locally compact spaces.

THEOREM 3.3. Consider any lattice space $\langle X, \mathscr{L}\rangle$. If $\mathscr{L}$ is $\delta$ and countably compact, then every element of $M_{\mathrm{R}}^{\sigma}(\mathscr{L})$ is perfect.

Proof. Assume $\mathscr{L}$ is $\delta$ and countably compact. Consider any element $\mu$ of $M_{\mathrm{R}}^{\sigma}(\mathscr{L})$. To show $\mu$ is perfect, use the relevant definition, namely, consider any function $f$ from $X$ to $\mathrm{R}$, such that $f$ is $\sigma(\mathscr{L})$-measurable, and any subset $E$ of $\mathbf{R}$, such that $f^{-1}(E) \in \sigma(\mathscr{L})$; show there exists a Borel set $B$ of $\mathbf{R}$, such that $B \subset E$ and $\mu\left(f^{-1}(B)\right)=\mu\left(f^{-1}(E)\right)$. Note that to show there exists a Borel set $B$ of $\mathrm{R}$, such that $B \subset E$ and $\mu\left(f^{-1}(B)\right)=\mu\left(f^{-1}(E)\right)$, it suffices to show that for every positive number $\epsilon$, there exists an R-closed set $F$ such that $F \subset E$ and $\mu\left(f^{-1}(F)\right)>\mu\left(f^{-1}(E)\right)-\epsilon$. Accordingly, consider any positive number $\epsilon$.

Now, use Lemma 3.2 as follows: consider $f^{-1}(E)$. Further, consider $\left.f\right|_{f^{-1}(E)}$ and denote it by $g$. Note since $f$ is $\sigma(\mathscr{L})$-measurable and $f^{-1}(E) \in \sigma(\mathscr{L}), g$ is $\sigma(\mathscr{L})$-measurable.

Thus $\mathscr{L}$ is $\delta, \mu \in M_{\mathrm{R}}^{\sigma}(\mathscr{L}), f^{-1}(E)$ is a $\sigma(\mathscr{L})$-measurable set, $g$ is a $\sigma(\mathscr{L})$ measurable function from $f^{-1}(E)$ to $\mathrm{R}$, and $\epsilon$ is a positive number. Then, by Lemma 3.2, there exists an element $D \in \mathscr{L}$ such that $D \subset f^{-1}(E),\left.g\right|_{D} \in$ $C_{b}(\mathscr{L})$ and $\mu\left(f^{-1}(E)-D\right)<\epsilon$. Consider any such $D$. Then $g(D)$ is R-closed (since $\mathscr{L}$ is countably compact), $g(D) \subset E$ and $\mu\left(f^{-1}(g(D))\right)>$ $\mu\left(f^{-1}(E)\right)-\epsilon$. Consequently $\mu$ is perfect.

NOTE. If $\mathscr{L}$ is countably compact, then $M_{\mathrm{R}}(\mathscr{L})=M_{\mathrm{R}}^{\sigma}(\mathscr{L})$. Consequently if $\mathscr{L}$ is $\delta$ and countably compact, then every element of $M_{\mathrm{R}}(\mathscr{L})$ is perfect.

APPLICATION 3.4. Consider any lattice space $\langle X, \mathscr{L}\rangle$. Denote the class of $\mathscr{L}$ compact sets by $\mathscr{K}$. Assume $\mathscr{K} \subset t \mathscr{L}$. (Note if $\mathscr{L}$ is (separating, disjunctive, and normal) or $T_{2}$, then $\mathscr{K} \subset t \mathscr{L}$ ). Consider $\mathscr{K} \cup\{X\}$ and denote it by $\mathscr{H}$. Note that $\mathscr{H}$ is a lattice, and that $\mathscr{H}$ is $\delta$ and countably compact. Hence, by Theorem 3.3, every element of $M_{\mathrm{R}}(\mathscr{H})$ is perfect. 
APPLICATION 3.5. Consider any topological space $X$ which is $T_{2}$, locally compact, $\sigma$-compact, and satisfies the Second Axiom of Countability. Now, consider any element of $M^{\sigma}(\mathscr{F})$, that is, any Borel measure $\mu$ of $X$. Denote the class of $\mathscr{F}$-compact sets by $\mathscr{K}$ and the class of those elements of $\mathscr{K}$ which are $G_{\delta}, s$ by $\mathscr{K}_{0}$. Set $\mathscr{K} \cup\{X\}=\mathscr{H}$. Note since $X$ is $T_{2}, \mathscr{H} \subset \mathscr{F}$. Furthermore, since $X$ satisfies the Second Axiom of Countability, $\mathscr{K}=\mathscr{K}_{0}$. Consequently $\mathscr{H}=\mathscr{K}_{0} \cup\{X\}$. Now, consider $\left.\mu\right|_{X(\mathscr{H})}$ and denote it by $\nu$. Thus $v \in M^{\sigma}(\mathscr{H})$. Note that $\mathscr{H}$ is $\delta$, and since $X$ is $\sigma$-compact, $\sigma(\mathscr{H})=\rho(\mathscr{H})$. Consequently $v \in M_{\mathrm{R}}^{\sigma}(\mathscr{H})$. Then, by Theorem 3.3, $v$ is perfect.

APPLICATION 3.6. Consider any topological space $X$ such that $X$ is countably compact, and let $\mathscr{L}=\mathscr{F}$. Then, by Theorem 3.3 , every element of $M_{\mathrm{R}}(\mathscr{F})$, that is, every regular, finite, Borel measure of $X$ is perfect.

APPLICATION 3.7. Consider any topological space $X$ such that $X$ is $T_{3 \frac{1}{2}}$ and pseudocompact, and let $\mathscr{L}=\mathscr{Z}$. Note $\mathscr{Z}$ is $\delta$ and countably compact. Further, note since $\mathscr{Z}$ is $\delta$ and $\sigma(\mathscr{Z})=\rho(\mathscr{Z}), M^{\sigma}(\mathscr{Z}) \subset M_{\mathrm{R}}^{\sigma}(\mathscr{Z})$. Then, by Theorem 3.3, every element of $M^{\sigma}(\mathscr{Z})$, that is, every finite Baire measure of $X$ is perfect.

\section{Lattice-measure tightness and lattice-measure perfectness}

In this section, the relationship between lattice-measure tightness and latticemeasure perfectness is investigated and applications are given whenever an opportunity arises.

The following theorem extends a result of Sazonov on topological spaces [9] to lattice spaces.

THEOREM 4.1. Consider any lattice space $\langle X, \mathscr{L}\rangle$. Let $\mu \in M^{\sigma}(\mathscr{L})$. (Note if $\mu \in M_{\mathrm{R}}^{t}(\mathscr{L})$, then $\mu \in M_{\mathrm{R}}^{\sigma}(\mathscr{L})$ ). If $\mathscr{L}$ is $\delta$ and $\mu \in M_{\mathrm{R}}^{t}(\mathscr{L})$, then $\mu$ is perfect.

PROOF. Assume $\mathscr{L}$ is $\delta$ and $\mu \in M_{\mathrm{R}}^{t}(\mathscr{L})$. To show $\mu$ is perfect, use (Lemma 2.2, Note), namely, show condition (**) is satisfied. Accordingly, consider any $\sigma(\mathscr{L})$-measurable function $f$ from $X$ to $\mathrm{R}$, and any positive number $\epsilon$, and show there exists an R-closed set $F$, such that $F \subset f(X)$ and $\mu\left(f^{-1}(F)\right)>\mu(X)-\epsilon$. 
$(\alpha)$ Note since $\mathscr{L}$ is $\delta, \mu \in M_{\mathrm{R}}^{\sigma}(\mathscr{L}), X$ is a $\sigma(\mathscr{L})$-measurable set, $f$ is a $\sigma(\mathscr{L})$-measurable function from $X$ to $\mathrm{R}$, and $\epsilon$ is a positive number, by Lemma 3.2, there exists an element $D \in \mathscr{L}$ such that $D \subset X,\left.f\right|_{D} \in C_{b}(\mathscr{L})$ and $\mu(X-D)<\epsilon / 2$. Consider any such $D$.

$(\beta)$ Note since $\mu \in M^{t}(\mathscr{L})$ and $\epsilon$ is a positive number, by the relevant definition, there exists an $\mathscr{L}$-compact set $K$ such that $\mu_{*}(X-K)<\epsilon / 2$. Consider any such $K$.

$(\gamma)$ Consider $K \cap D$. Then $f(K \cap D)$ is R-closed, $f(K \cap D) \subset f(X)$ and $\mu\left(f^{-1}(f(K \cap D))\right)>\mu(X)-\epsilon$. Consequently $\mu$ is perfect.

Corollary 4.2. Consider any lattice space $\langle X, \mathscr{L}\rangle$. Let $\mu \in M^{\sigma}(\mathscr{L})$. If $\mathscr{L}$ is $\delta$ and strongly measure replete, then $\mu \in M_{\mathrm{R}}^{\sigma}(\mathscr{L})$ implies $\mu$ is perfect.

PROOF. Assume $\mathscr{L}$ is $\delta$ and strongly measure replete. Now, assume $\mu \in$ $M_{\mathrm{R}}^{\sigma}(\mathscr{L})$. Note that since $\mathscr{L}$ is strongly measure replete, by definition, $M_{\mathrm{R}}^{\sigma}(\mathscr{L}) \subset$ $M_{\mathrm{R}}^{t}(\mathscr{L})$. Consequently $\mu \in M_{\mathrm{R}}^{t}(\mathscr{L})$. Thus $\mathscr{L}$ is $\delta$ and $\mu \in M_{\mathrm{R}}^{t}(\mathscr{L})$. Hence, by Theorem $4.1, \mu$ is perfect.

APPLICATION 4.3. Consider any topological space $X$ which is $T_{2}$, locally compact, and Lindelöf. Since $X$ is $T_{2}$, locally compact, and Lindelöf, $\mathscr{F}$ is strongly measure replete [3]. Then, by Corollary 4.2, every element of $M_{\mathrm{R}}^{\sigma}(\mathscr{F})$, that is, every regular, finite, Borel measure of $X$, is perfect.

APPLICATION 4.4. Consider any topological space $X$ which is complete, separable, and metrizable. Since $X$ is metrizable, $M^{\sigma}(\mathscr{F})=M_{\mathrm{R}}^{\sigma}(\mathscr{F})$, and since $X$ is complete, separable, and metrizable, $\mathscr{F}$ is strongly measure replete [8]. Then, by Corollary 4.2, every element of $M^{\sigma}(\mathscr{F})$, that is, every finite Borel measure of $X$, is perfect.

APPLICATION 4.5. Consider the topological space $\langle\mathbf{R}, \mathscr{T}\rangle$ where $\mathscr{T}$ is the usual topology. Note that the topological space $\mathrm{R}$ is complete, separable and metrizable. Hence, by Application 4.4, every finite Borel measure of $R$ is perfect.

Next, the question of when lattice-measure perfectness implies lattice-measure tightness is investigated.

LEMMA 4.6. Consider any set $X$ and denote its general element by $x$. Furthermore, consider any sequence $\left\langle E_{n}\right\rangle$ in $\mathscr{P}(X)$, and its characteristic function $h[11]$. Finally, consider the class of Borel sets of $R$ and denote it by $\mathscr{B}$. Then 
(i) $\sigma\left(\left\{E_{n} ; n \in N\right\}\right)=h^{-1}(\mathscr{B})$.

(ii) $h$ is one-to-one if and only if for every two values $x_{1}, x_{2}$ of $x$, such that $x_{1} \neq x_{2}$, there exists a value $m$ of $n$, such that $x_{1} \in E_{m}$ and $x_{2} \notin E_{m}$, or $x_{1} \notin E_{m}$ and $x_{2} \in E_{m}$.

REMARK. This fact is known [11].

LEMMA 4.7. Consider the following: any topological space $X$ which is $T_{1}$ and satisfies the Second Axiom of Countability, any countable base for $X$, and the characteristic function $h$ of $\left\langle U_{n}\right\rangle$. Then $h$ is one-to-one (or, equivalently, $h^{-1}$ is a function) and $h^{-1}$ is continuous.

(Proof omitted).

LEMMA 4.8. Consider the setting of the definition of lattice-perfect measure. Let $\mu \in M^{\sigma}\left(\mathscr{L}_{1}\right)$. If $\mathscr{L}_{2}$ is complement generated $\left(\right.$ or $\mathscr{L}_{2}$ is $\delta$ and $\sigma\left(\mathscr{L}_{2}\right) \subset$ $\left.s\left(\mathscr{L}_{2}\right)\right)$, then $\mu$ is $\left\langle\mathscr{L}_{1}, \mathscr{L}_{2}\right\rangle$-perfect if and only if for every $\left\langle\sigma\left(\mathscr{L}_{1}\right), \sigma\left(\mathscr{L}_{2}\right)\right\rangle$ measurable function $T$ from $X$ to $Y$, for every subset $E$ of $Y$, such that $T^{-1}(E) \in$ $\sigma\left(\mathscr{L}_{1}\right)$, and for every positive number $\epsilon$, there exists an element $L_{2} \in \mathscr{L}_{2}$ such that $L_{2} \subset E$ and $\mu T^{-1}\left(L_{2}\right)>\mu T^{-1}(E)-\epsilon$.

(Proof omitted).

THEOREM 4.9. Consider any topological space $\left\langle X, \mathscr{F}_{1}\right\rangle$ and the topological space $\left\langle R, \mathscr{F}_{2}\right\rangle$ where $\mathscr{F}_{2}$ is the usual topology. If $\mathscr{F}_{1}$ is $T_{1}$ and $\mathscr{F}_{1}$ satisfies the Second Axiom of Countability, then for every element $\mu \in M^{\sigma}\left(\mathscr{F}_{1}\right)$, if $\mu$ is $\left\langle\mathscr{F}_{1}, \mathscr{F}_{2}\right\rangle$-perfect, then $\mu$ is $\mathscr{F}_{1}$-tight.

Proof. Assume $\mathscr{F}_{1}$ is $T_{1}$ and $\mathscr{F}_{1}$ satisfies the Second Axiom of Countability. Now, consider the following: any countable base $\mathscr{F}_{1}^{*}$ for $\mathscr{F}_{1}$, using the assumption; the characteristic function $h$ of $\mathscr{F}_{1}^{*}, h(X)$, and $h(X) \cap \mathscr{F}_{2}$; any element $\mu \in M^{\sigma}\left(\mathscr{F}_{1}\right)$ such that $\mu$ is $\left\langle\mathscr{F}_{1}, \mathscr{F}_{2}\right\rangle$-perfect. To show $\mu$ is $\mathscr{F}_{1}$-tight, use the relevant definition, namely, consider any positive number $\epsilon$ and show there exists an $\mathscr{F}_{1}$-compact set $K_{1}$ such that $\mu_{*}\left(X-K_{1}\right)<\epsilon$ or, equivalently, $\mu^{*}\left(K_{1}\right)>\mu(X)-\epsilon$. Note that since $\mu$ is $\left\langle\mathscr{F}_{1}, \mathscr{F}_{2}\right\rangle$-perfect and $\mathscr{F}_{2}$ is complement generated (or $\sigma\left(\mathscr{F}_{2}\right) \subset s\left(\mathscr{F}_{2}\right)$ ), by Lemma 4.8 , for every $\left\langle\sigma\left(\mathscr{F}_{1}\right), \sigma\left(\mathscr{F}_{2}\right)\right\rangle$-measurable function $T$ from $X$ to $\mathrm{R}$, and for every subset $E$ of $\mathrm{R}$, such that $T^{-1}(E) \in \sigma\left(\mathscr{F}_{1}\right)$, there exists an element $F_{2} \in \mathscr{F}_{2}$ such that $F_{2} \subset E$ and $\mu\left(T^{-1}\left(F_{2}\right)\right)>\mu\left(T^{-1}(E)\right)-\epsilon$. Now, by Lemma 4.6, part (i), $h$ is $\left\langle\sigma\left(\mathscr{F}_{1}\right), \sigma\left(\mathscr{F}_{2}\right)\right\rangle^{\dagger}$-measurable. Consequently there exists an element $F_{2} \in \mathscr{F}_{2}$ 
such that $F_{2} \subset h(X)$ and $\mu\left(h^{-1}\left(F_{2}\right)\right)>\mu\left(h^{-1}(h(X))\right)-\epsilon(=\mu(X)-\epsilon)$. Consider any such $F_{2}$. Note that since $F_{2} \subset h(X) \subset[0,1], F_{2}$ is $\mathscr{F}_{2}$-compact. Consequently $F_{2}$ is $h(X) \cap \mathscr{F}_{2}$-compact. Furthermore, since $\mathscr{F}_{1}$ is $T_{1}$ and $\mathscr{F}_{1}$ satisfies the Second Axiom of Countability, $h^{-1}$ is a function and $h^{-1}$ is continuous (Lemma 4.7). Consequently $h^{-1}\left(F_{2}\right)$ is $\mathscr{F}_{1}$-compact. Thus $h^{-1}\left(F_{2}\right)$ is $\mathscr{F}_{1}$-compact and $\mu\left(h^{-1}\left(F_{2}\right)\right)>\mu(X)-\epsilon$. Hence $\mu$ is $\mathscr{F}_{1}$-tight.

Theorems 4.1 and 4.9 yield the following.

COROLLARY 4.10. If $\mathscr{F}_{1}$ is $T_{1}$ and $\mathscr{F}_{1}$ is complement generated or $\sigma\left(\mathscr{F}_{1}\right) \subset$ $s\left(\mathscr{F}_{1}\right)$, and $\mathscr{F}_{1}$ satisfies the Second Axiom of Countability, then for every element $\mu \in M^{\sigma}\left(\mathscr{F}_{1}\right), \mu \in M_{\mathrm{R}}^{t}\left(\mathscr{F}_{1}\right)$ if and only if $\mu$ is $\left\langle\mathscr{F}_{1}, \mathscr{F}_{2}\right\rangle$-perfect, that is, a finite Borel measure of $X$ is tight if and only if it is perfect.

REMARK. Sazonov calls a regular tight measure dense.

Corollary 4.10 is a generalization of [9, page 250 , Theorem 11$]$.

\section{Acknowledgement}

The author wishes to express his appreciation to Long Island University for partial support of the present work through a grant of released time from teaching duties.

\section{References}

[1] A. D. Alexandroff, 'Additive set functions in abstract spaces', Mat. Sb. 51 (1941), 563-628.

[2] G. Bachman and A. Sultan, 'Extensions of regular lattice measures with topological applications', J. Math. Anal. Appl. 57 (1977), 539-559.

[3] G. Bachman and M. Szeto, 'On strongly measure replete lattices and the general Wallman remainder', Fund. Math. 122 (1984), 199-217.

[4] Z. Frolik, 'Prime filters with the c.i.p.', Comment. Math. Univ. Carolin. 13 (1972), 553-575.

[5] B. V. Gnedenko and A. N. Kolmogoroff, Limit distributions for sums of independent random variables (GITTL, Moscow, 1949) (in Russian); (English translation: Addison Wesley, Cambridge, Mass., 1954).

[6] P. Grassi, 'On subspaces of replete and measure replete spaces', Canad. Math. Bull. 27 (1984), 58-64.

[7] G. Nöbeling, Grundlagen der analytischen Topologie (Springer-Verlag, Berlin, 1954). 
[8] K. Parthasarathy, Probability measures on metric spaces (Academic Press, New York, London, 1967).

[9] V. V. Sazonov, 'On perfect measures', Amer. Math. Soc. Transl. Ser. 248 (1965), 229-254.

[10] M. Szeto, 'Measure repleteness and mapping preservations', J. Indian Math. Soc. 43 (1979), 35-52.

[11] E. Szpilrajn, 'The characteristic function of a sequence of sets and some of its applications', Fund. Math. 31 (1938), 207-223.

[12] H. Wallman, 'Lattices and topological spaces', Ann. of Math. 39 (1938), 112-126.

Long Island University

Brooklyn, N.Y. 11201

USA 